\title{
TRANSIENT ELECTROMAGNETIC RESPONSE OF A LAYERED CONDUCTING MEDIUM AT ASYMPTOTICALLY LATE TIMES
}

\author{
D. M. O'BRIEN ${ }^{1}$ AND R. S. SMITH ${ }^{2}$
}

(Received 25 November 1983; revised 21 May 1984)

\begin{abstract}
In this paper we consider a pair of horizontal conducting loops in the air above a horizontally layered ground. The transmitting loop is driven by a current source which rises from zero at time zero to a final constant value at time $\tau$. We first compute the e.m.f. induced in the receiving loop and derive an asymptotic series for the e.m.f. at late times. Secondly, we estimate the error in truncating the asymptotic series at $N$ terms and design a reliable numerical algorithm for summing the asymptotic series.
\end{abstract}

\section{Introduction}

In this paper we consider the transient electromagnetic (TEM) response of a horizontally layered medium such as that shown in Figure 1, where the conductivities are independent of frequency and all layers have the same magnetic permeability. This subject has been studied by many authors, in particular, by authors interested in the application of electromagnetic techniques to the detection of minerals. An excellent guide to the literature is contained in the book on geoelectromagnetism published recently by Wait [22]. In principle, the TEM response is completely known, because the electromagnetic Green's tensor for a layered conducting medium reduces to an elementary expression after Laplace (or Fourier) transformation of the time variable and Hankel transformation of the

\footnotetext{
${ }^{1}$ CSIRO Division of Atmospheric Research, Private Bag 1, Mordialloc, Vic. 3195, Australia.

${ }^{2}$ Department of Physics, Univerisity of Toronto, Toronto, Ontario, Canada M5S 1A7.

(1) Copyright Australian Mathematical Society 1985, Serial-fee code 0334-2700/85
} 


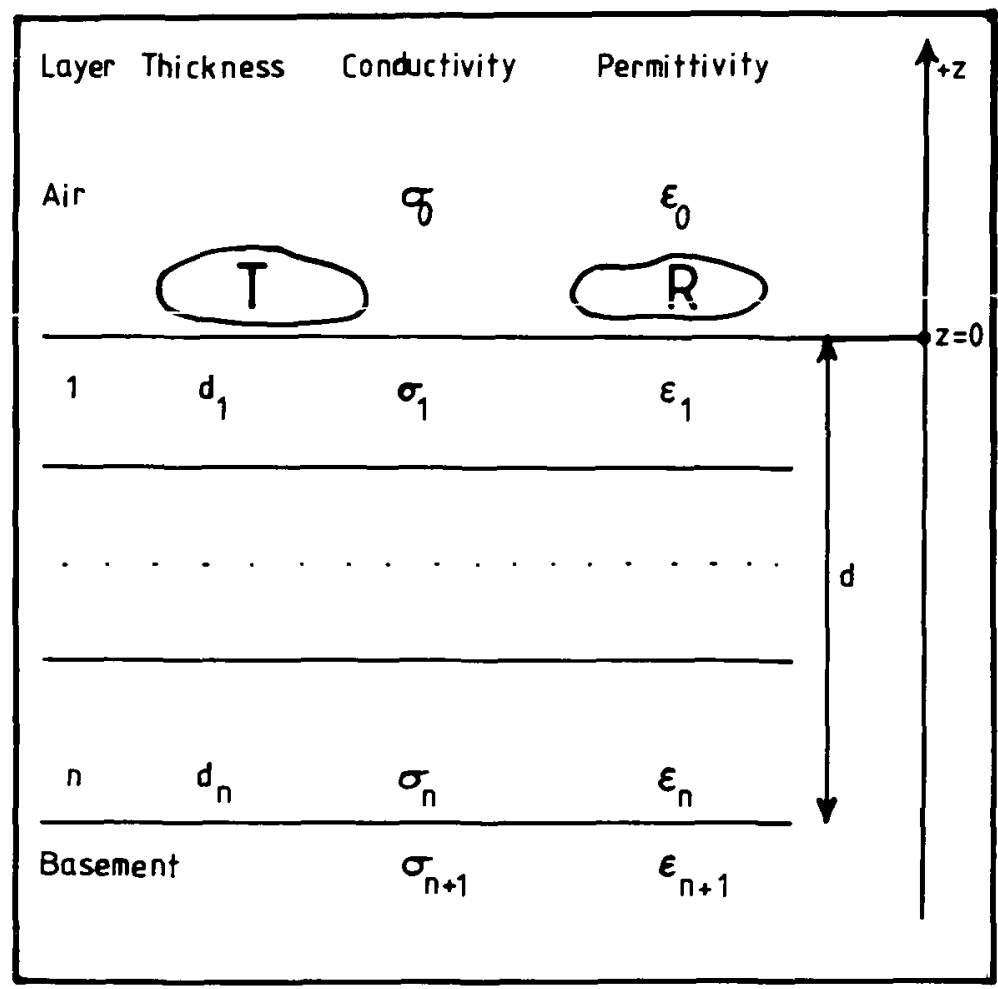

Figure 1

racial cylindrical coordinate, but in practice the numerical inversion of the two integral transforms poses a problem. Morrison, Phillips and O'Brien [16] gave an early solution which used the trapezoidal quadrature rule for the inverse Hankel transform and the fast Fourier algorithm for the inverse Fourier transform. After the development of digital filters for the Hankel transform of arbitrary order $\nu$, several new programs were written which used digital filters for the Hankel transform and possibly also for the Fourier transform, since the latter can be formulated as a Hankel transform with order $\nu=\frac{1}{2}$. These programs were considerably more efficient. (Koefoed [10], Mallick and Verma [15], Anderson [2].) Knight and Raiche [9] made a further significant improvement in efficiency by reversing the order of the inverse integral transforms, and applied the Gaver-Stehfest $[6,19,20]$ algorithm to the inverse Laplace transform followed by an adaptive version of Patterson's [17] algorithm for Gaussian quadrature to evaluate the Hankel transform. The success of Knight and Raiche's strategy is due to the gain in speed and accuracy of the Gaver-Stehfest algorithm when applied to functions known analytically rather than numerically. After publication of their paper, Knight and Raiche replaced Patterson's integration with a digital filter, and their modified algorithm is currently the best available. 
Complementary to these computer programs, which attempt to invert the integral transforms numerically, is the work on asymptotic expansions of the TEM response at both early and late times. Lee and Lewis [14] established that at early or late times the TEM response approaches that of a half space whose conductivity is equal to that of the top layer or the bottom layer, respectively. Lee [13] also developed a two term asymptotic expansion valid for late times, which subsumed earlier results by Kamenetski [7] and Kaufman [8]. In addition to their simplicity and speed, the asymptotic expansions often have the useful property that they work best when the more general computer programs are most costly. For example, Knight and Raiche's algorithm seems to be slowest at late times, precisely when Lee's asymptotic formula is applicable.

If the object were to compute the TEM response of a single layered medium, then many of the refinements above would not be necessary, because even the most basic algorithm will obtain the TEM response for relatively little cost. However, in practice we want to infer the structure of a layered ground from observations of its TEM response, and to do so we employ an optimisation algorithm which adjusts the model in order to minimise a cost function which measures the discrepancy between the actual data and the data predicted by the model. The optimisation algorithm will be iterative and will proceed from an initial guess for the model to a final 'solution', which is a local minimiser of the cost function. The 'solution' is dependent upon the initial guess, so, in addition to all the iterations of the optimisation algorithm, it is essential to experiment with different initial guesses. If all this calculation is to be performed in real time, so that a geophysicist can immediately interpret his survey data, then the computer code which calculates the TEM response of the layered ground must employ every known time saving device.

With this motivation, we now turn to the content of this paper.

We develop a late time asymptotic expansion for the TEM response of the layered conducting ground depicted in Figure 1, and we establish the region of usefulness of the expansion. The terms of the expansion are easily computed and can be programmed on almost any microcomputer. The expansion may be used on its own for a very rapid first interpretation of field data, or may be coupled with existing software such as the TEM program of Knight and Raiche [9], to produce faster inversion programs.

The transmitting and receiving loops must be horizontal, but otherwise their shapes and positions are arbitrary, although in practice we will require the loops to be either circular or rectangular. In particular, the loops may be either coincident or distinct. We assume that the transmitting loop carries a current density

$$
\mathbf{J}(\mathbf{x}, t)=\mathbf{M}(\mathbf{x}) I(t)
$$


where $\mathbf{M}$ is horizontal, divergence free and has compact support, and $I$ is the ramp function

$$
I(t)= \begin{cases}0 & \text { if } t<0, \\ t / \tau & \text { if } 0 \leqslant t \leqslant \tau, \\ 1 & \text { if } \tau<t .\end{cases}
$$

Such a temporal variation models the UTEM prospecting system (Lamontagne, Lodha, Macnae and West [12]), the PEM prospecting system (Crone [5]), and also models SIROTEM (Buselli and O'Neill [3]) in the limit $\tau \rightarrow 0$.

It is worth noting that the extension of the analysis to receiving loops which are not horizontal is straightforward, and only requires the introduction of a new loop function. However, the extension to transmitters other than horizontal loops requires more substantial changes. For such sources two complications arise: firstly, the electric field intensity has a nonzero vertical component which is discontinuous across the interfaces between the layers; and secondly, a tensor rather than scalar Green's function is needed. Nevertheless, the analysis is the same in principle, although more cumbersome in practice.

We will work exclusively with variables scaled as follows:

$$
\begin{array}{lll}
\text { coordinates } & \mathbf{x} \rightarrow \mathbf{x} / l & \text { (dimensionless); } \\
\text { conductivity } & \sigma \rightarrow \sigma / \sigma_{n+1} & \text { (dimensionless); } \\
\text { time } & t \rightarrow t /\left(l^{2} \mu \sigma_{n+1}\right), & \text { (dimensionless); } \\
\text { permittivity } & \varepsilon \rightarrow \varepsilon /\left(l^{2} \mu \sigma_{n+1}^{2}\right), & \text { (dimensionless); } \\
\text { electric field } & \mathbf{E} \rightarrow \mathbf{E} l & \text { (volts/unit scaled length); } \\
\text { current density } & \mathbf{J} \rightarrow \mathbf{J} l^{2} & \text { (amps./unit scaled area). }
\end{array}
$$

Here $l$ denotes any typical length chosen as the unit of length, such as the radius or side length of the transmitting loop. We will compute the dimensionless quantity

$$
Z(t)=l \sigma_{n+1} V / I_{p},
$$

where $V$ is the e.m.f. induced in the receiving loop, measured in volts, and $I_{p}$ is the peak value of the current in the transmitting loop, measured in amps. $Z(t)$ is clearly related to the mutual impedance of the transmitting and receiving loops over the layered medium.

The key to the asymptotic analysis of the TEM response lies in the treatment of the inverse Laplace transform. We examine the singularities of the function to be transformed and show that the contour of integration may be deformed around a cut along the negative real axis. Then $Z(t)$ has the asymptotic form

$$
Z(t) \sim\left[B_{1}(t)-B_{1}(t-\tau)\right] / \tau,
$$


where

$$
B_{i}(t)=\pi^{-2} \int_{0}^{\infty} d x \exp \left(-x^{2} t\right) x^{2(i-1)} S(x)
$$

and

$$
S(x)=x^{2} \int_{0}^{1} d m\left(1-m^{2}\right)^{1 / 2} m P(m x) / Q(m, x) .
$$

Here $P$ is a function which we call the loop function, because it depends solely on the disposition of the loops and not on the structure of the layered medium, whereas the function $Q$ has the opposite dependence. Watson's lemma (Copson [4]) can be applied to $B_{i}(t)$ and we obtain the asymptotic expansion

$$
B_{i}(t) \sim A_{1}(t)=\left(2 \pi^{2}\right)^{-1} \sum_{r=0}^{\infty} S_{r} \Gamma((r+1) / 2+i) t^{-((r+1) / 2+1)},
$$

where $S_{0}, S_{1}, \ldots$ are the coefficients in a power series for $S$ convergent for small $x$ :

$$
S(x)=x^{2} \sum_{r=0}^{\infty} S_{r} x^{r} .
$$

The coefficients $S_{0}, S_{1}, \ldots$ depend upon both the geometry of the loops and the structure of the medium, but are independent of time. Consequently, the coefficients only need be computed once if the mutual impedance is required at several times. If the time variation of the source current is a step rather than a ramp, then the appropriate asymptotic expansion is obtained in the limit as $\tau \rightarrow 0$, namely

$$
Z(t) \sim-A_{2}(t) \text {. }
$$

Listed in Table 1 are the times taken to compute the coefficients by the CYBER 173 machine at the University of Adelaide.

TABLE 1

\begin{tabular}{|c|c|}
\hline Number of Layers & Time to compute $S_{0}, \ldots, S_{15}$ \\
\hline 1 & 36 msec. \\
2 & 49 msec. \\
3 & $66 \mathrm{msec}$. \\
4 & $99 \mathrm{msec}$. \\
5 & $181 \mathrm{msec}$. \\
\hline
\end{tabular}

The extra time required to sum the asymptotic series is negligible, approximately 1 msec per time.

In all practical prospecting systems, the quantity that is actually measured is not the mutual impedance at a particular time, but rather the average of this quantity over a time channel

$$
t_{0}<t<t_{1}
$$


Thus, the observed quantity is

$$
Z\left(t_{0}, t_{1}\right)=\left(t_{1}-t_{0}\right)^{-1} \int_{t_{0}}^{t_{1}} d t Z(t)
$$

Provided that both $t_{0}$ and $t_{1}$ are sufficiently late, then the asymptotic expansion may be substituted for $Z(t)$ and integrated termwise to give

$$
Z\left(t_{0}, t_{1}\right)= \begin{cases}\frac{A_{0}\left(t_{0}\right)-A_{0}\left(t_{1}\right)-A_{0}\left(t_{0}-\tau\right)+A_{0}\left(t_{1}-\tau\right)}{\tau\left(t_{1}-t_{0}\right)}, & \tau>0, \\ \frac{A_{1}\left(t_{1}\right)-A_{1}\left(t_{0}\right)}{\left(t_{1}-t_{0}\right)}, & \tau=0 .\end{cases}
$$

The asymptotic series in (1.7) above is divergent (except for the degenerate case of a half space without overburden), but, for any fixed number of terms, will provide any desired accuracy for sufficiently late times. However, before the asymptotic series can be used successfully, the problems of choosing the number of terms and of deciding how late is 'sufficiently late' must be addressed. We will show that

$$
\left|B_{i}(t)-B_{i}^{N}(t)\right|<K \xi^{2(i+1)} F\left(N+2(i+1), \xi t^{1 / 2}\right),
$$

where $B_{1}^{N}$ is the sum to $N$ terms of the asymptotic series and

$$
F(m, z)=\Gamma(m / 2) / z^{m} \text {. }
$$

The number $\xi$ is computable exactly in principle, but in practice is given sufficiently accurately by the following expression

$$
\xi^{-1}= \begin{cases}d\left|\theta_{1}\right|\left(1+|x|^{1 / 2}\right), & x \leqslant 0 \\ d\left|\theta_{1}\right|(1+\chi)^{1 / 2}, & x \geqslant 0\end{cases}
$$

where

$$
\begin{aligned}
& \chi=\left(\theta_{1}+\theta_{2}\right) / \theta_{1}^{2}, \\
& \theta_{1}=d^{-1} \sum_{i=1}^{n}\left(1-\sigma_{i}\right) d_{i}, \\
& \theta_{2}=d^{-2} \sum_{i=1}^{n}\left(1-\sigma_{t}\right) d_{i}\left(d_{1}+\cdots+d_{t-1}-d_{i+1}-\cdots-d_{n}\right)
\end{aligned}
$$

and finally

$$
d=\sum_{i=1}^{n} d_{i}
$$


If $\xi^{2} t>1$, then the bound, regarded as a function of $N$, decreases at first, passes through a minimum, and then diverges. If $\xi^{2} t \leqslant 1$, the bound simply diverges. The minimum of $F(m, z)$ occurs when

$$
\psi(m / 2)=2 \log z,
$$

where $\psi$ is the psi function, and typical values of $F$ at the minimum are listed in Table 2 .

TABLE 2

\begin{tabular}{|c|c|c|}
\hline$z$ & $m$ at minimum & $F(m, z)$ at the minimum \\
\hline 2 & 9 & $2.27 \times 10^{-2}$ \\
3 & 19 & $1.03 \times 10^{-4}$ \\
4 & 33 & $7.03 \times 10^{-8}$ \\
5 & 51 & $6.95 \times 10^{-12}$ \\
\hline
\end{tabular}

On the basis of these results, a reasonable strategy for truncating the asymptotic series might be as follows.

(1) Compute $\chi$ and $\xi$ from formulae (1.14) and (1.13).

(2) Check whether

$$
\xi^{2} t>3 \text {. }
$$

If not, set a warning flag to indicate that the time is too early.

(3) Find the closest integer $N$ to the solution of (1.16). This is easily done as the values of the psi function can be generated by recursion and then stored.

(4) Sum the asymptotic series to $N$ terms.

We implemented this strategy and found it successful but too conservative, no doubt because the estimate (1.12) is not sufficiently sharp. Instead we adopted the following pragmatic strategy.

(1) Compute a running average of the moduli of two terms.

(2) Choose $N$ corresponding to the minimum of the running average.

(3) Take the average of the moduli of the last term and the first omitted term as a measure of the error.

We found the running average necessary because the odd and even order terms occasionally had different orders of magnitude. The success of the strategy is shown in Figure 2 where we plot contours of the error for a medium with a single conductive layer with unit thickness above the basement. The prospecting system is SIROTEM with coincident circular loops with unit radius and a step function current source driving the transmitter loop. The variables in the plot are time and the conductivity of the layer. The errors were computed by comparison of the asymptotic series with the program of Knight and Raiche [9]. Also shown on the 
plot are the theoretical curve

$$
\xi^{2} t=3
$$

and the curve along which the pragmatic algorithm believes it has achieved an accuracy of $1 \%$. The algorithm clearly is reliable.

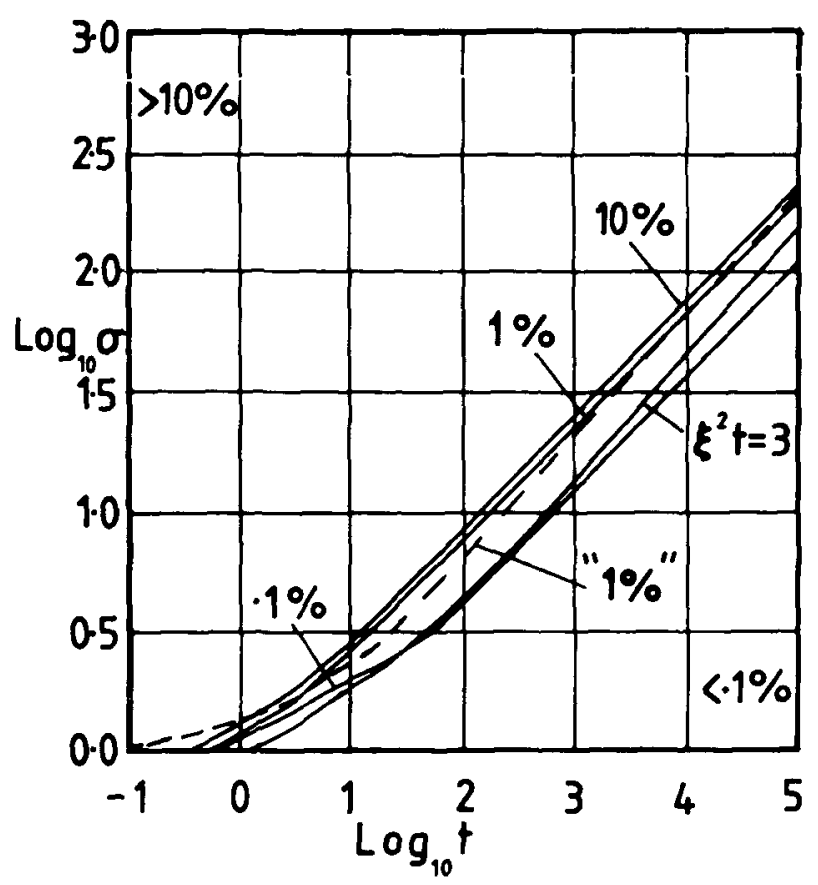

Figure 2

\section{Derivation of $Z(s)$}

We begin the analysis with the following expressions for the Laplace transform of the e.m.f. induced in the receiving loop:

$$
\begin{gathered}
V(s)=\int_{R} d \mathbf{x} \cdot \mathbf{E}(\mathbf{x}, s), \\
\mathbf{E}(\mathbf{x}, s)=\frac{-s}{l \sigma_{n+1}} \int_{T} d^{3} x^{\prime} g\left(\mathbf{x}, \mathbf{x}^{\prime}, s\right) \mathbf{J}\left(\mathbf{x}^{\prime}, s\right),
\end{gathered}
$$

and

$$
\begin{aligned}
g\left(\mathbf{x}, \mathbf{x}^{\prime}, s\right)=(4 \pi)^{-1} \int_{0}^{\infty} d \lambda\left(\lambda / k_{0}\right) J_{0}\left(\lambda|\mathbf{r}-\mathbf{r}|^{\prime} \mid\right) \\
\cdot\left[\exp \left(-k_{0}\left|z-z^{\prime}\right|\right)+\exp \left(-k_{0}\left(z+z^{\prime}\right)\right) b / a\right]
\end{aligned}
$$


Here $V$ and $\mathbf{E}$ denote the induced e.m.f. and the electric field intensity. The variables $k_{0}, k_{1}, \ldots, k_{n+1}$ are defined by

$$
k_{1}=\left(\varepsilon_{i} s^{2}+\sigma_{i} s+\lambda^{2}\right)^{1 / 2}
$$

and $a$ and $b$ are elements of the matrix $A$ defined as follows:

$$
\begin{aligned}
A & =\left[\begin{array}{ll}
a & b \\
c & d
\end{array}\right] \\
& =\left[\begin{array}{cc}
\exp \left(k_{n+1} d\right) & 0 \\
0 & \exp \left(-k_{n+1} d\right)
\end{array}\right]\left[\begin{array}{cc}
1 & k_{n+1} \\
1 & -k_{n+1}
\end{array}\right] T_{n} \cdots T_{1}\left[\begin{array}{cc}
1 & k_{0} \\
1 & -k_{0}
\end{array}\right]^{-1}
\end{aligned}
$$

where

$$
T_{\imath}=\left[\begin{array}{cc}
\cosh k_{\imath} d_{\imath} & k_{1} \sinh k_{\imath} d_{1} \\
k_{\imath}^{-1} \sinh k_{\imath} d_{i} & \cosh k_{\imath} d_{\imath}
\end{array}\right] \text {. }
$$

This result may be derived along the lines followed by Morrison, Phillips and O'Brien [16]. Alternatively, one can prove that the special properties of the source current density $\mathbf{J}$, that it be horizontal and divergence free, imply that there are no charge distributions on the interfaces between the layers and that Maxwell's equations reduce to the telegraph equation for a single scalar field. The telegraph equation separates in rectangular coordinates into ordinary differential operators, whose spectral kernels can be constructed by standard techniques. Convolution of these kernels yields the spectral kernel for the telegraph operator from which Green's function $g$ is constructed. The remaining steps which lead to $V$ are then trivial.

The function $g$ represents the effect at $\mathbf{x}$ of a source at $\mathbf{x}^{\prime}$ and contains geometrical optics terms which must be isolated and treated separately. To do so, let $\tilde{\mathbf{x}}^{\prime}$ represent the reflection of $\mathbf{x}^{\prime}$ in the plane $z=0$. Then

$$
g\left(\mathbf{x}, \mathbf{x}^{\prime}, s\right)=g_{0}\left(\left|\mathbf{x}-\mathbf{x}^{\prime}\right|, s\right)+p g_{0}\left(\left|\mathbf{x}-\tilde{\mathbf{x}}^{\prime}\right|, s\right)+f\left(\mathbf{x}, \mathbf{x}^{\prime}, s\right)
$$

where

$$
g_{0}(r, s)=\exp \left[-\left(\varepsilon_{0} s^{2}+\sigma_{0} s\right)^{1 / 2} r\right] /(4 \pi r)
$$

and

$$
f\left(\mathbf{x}, \mathbf{x}^{\prime}, s\right)=(4 \pi)^{-1} \int_{0}^{\infty} d \lambda\left(\lambda / k_{0}\right) J_{0}\left(\lambda\left|\mathbf{r}-\mathbf{r}^{\prime}\right|\right) \exp \left(-k_{0}\left(z+z^{\prime}\right)\right)[b / a-p]
$$

The first $g_{0}$ term is usually called the primary field in the geophysical TEM literature, and is the field that would remain if the earth were removed, whereas the second $g_{0}$ term is a reflection from the air-earth interface. The constant $p$ is 
ultimately fixed by boundary conditions, but its actual value will not concern us because it will cancel from the asymptotic expansion. The interpretation of $g$ becomes apparent after we compute its inverse Laplace transform:

$$
g_{0}(r, t)=\frac{\exp \left(-t / t_{0}\right)}{4 \pi r}\left[\delta(t-r / c)+\frac{H(t-r / c) r I_{1}\left(\left(t^{2}-r^{2} / c^{2}\right)^{1 / 2} / t_{0}\right)}{c t_{0}\left(t^{2}-r^{2} / c^{2}\right)^{1 / 2}}\right]
$$

where

$$
\begin{aligned}
t_{0} & =2 \varepsilon_{0} / \sigma_{0}, \\
c & =\varepsilon_{0}^{-1 / 2},
\end{aligned}
$$

and $H$ is the step function,

$$
H(t)= \begin{cases}0 & \text { if } t<0 \\ \frac{1}{2} & \text { if } t=0 \\ 1 & \text { if } t>0\end{cases}
$$

The case of most interest is that obtained by setting $\sigma_{0}=0$, so that the air is a perfect insulator, for then

$$
g_{0}(r, t)=\delta(t-r / c)(4 \pi r) .
$$

Here $c$ is the speed of light in air (in scaled variables!), so the first term in (2.7) represents the passage of a wave front directly from $\mathbf{x}$ to $\mathbf{x}^{\prime}$, whereas the second represents the wave front reflected from the interface between the air and the earth.

In the application to geoprospecting systems, the contributions from the $g_{0}$ terms occur at times too early to measure. Indeed, if $\mathbf{E}_{0}$ denotes the contribution to $\mathbf{E}$ from $g_{0}$, then

$$
\begin{aligned}
\mathbf{E}_{0}(\mathbf{x}, t) & =-\left(l \sigma_{n+1}\right)^{-1} \int_{0}^{t} d t^{\prime} \int_{r} d^{3} x^{\prime} g_{0}\left(r, t-t^{\prime}\right) \mathbf{j}\left(\mathbf{x}^{\prime}, t^{\prime}\right) \\
& =-\left(l \sigma_{n+1} \tau\right)^{-1} \int_{T} d^{3} x^{\prime} \mathbf{M}\left(\mathbf{x}^{\prime}\right) H(t-r / c) H(\tau-(t-r / c)) /(4 \pi r) .
\end{aligned}
$$

Since the speed of light in air is so large compared with the separation of the source and receiver, the terms involving $c$ may be dropped to give

$$
\mathbf{E}_{0}(\mathbf{x}, t)=-\left(l \sigma_{n+1} \tau\right)^{-1} H(\tau-t) \int_{T} d^{3} x^{\prime} \mathbf{M}\left(\mathbf{x}^{\prime}\right) /(4 \pi r) .
$$

Consequently, $\mathbf{E}_{0}$ vanishes for times later than $\tau$. Since our aim is to analyse the signal at late times, we may discard the $g_{0}$ terms throughout the rest of the paper. 
If we combine formulae (2.1), (2.2) and (2.3), we obtain

$$
Z(s)=-(4 \pi)^{-1} s I(s) \int_{0}^{\infty} d \lambda\left(\lambda / k_{0}\right) P(\lambda) \exp \left[-\left(k_{0}-\lambda\right)\left(z+z^{\prime}\right)\right][b / a-p]
$$

where

$$
P(\lambda)=\int_{R} d \mathbf{x} \cdot \int_{T} d^{3} x^{\prime} J_{0}\left(\lambda \mid \mathbf{r}-\mathbf{r}^{\prime}\right) \mathbf{M}\left(\mathbf{x}^{\prime}\right) \exp \left[-\lambda\left(z+z^{\prime}\right)\right] .
$$

We call $P$ the loop function, because all the geometric information concerning the disposition of the loops is embodied in $P$. So far we have not restricted $\mathbf{M}$, apart from requiring $\mathbf{M}$ to be horizontal and divergence free, but we will now impose the 'thin wire approximation' in which we assume that $\mathbf{M}$ is concentrated on the axis of the transmitting loop. Thus, we now let $T$ denote the axis of the transmitting loop, rather than its volume, and parametrise $T$ by the curve

$$
q \mapsto \mathbf{x}^{\prime}(q), \quad 0 \leqslant q \leqslant|T| .
$$

where $|T|$ is the circumference of $T$. Then $d \mathbf{x}^{\prime} / d q$ is a unit vector tangential to $T$ and

$$
\mathbf{M}=d \mathbf{x}^{\prime} / d q
$$

Hence,

$$
P(\lambda)=\int_{R} d \mathbf{x} \cdot \int_{T} d q J_{0}\left(\lambda\left|\mathbf{r}-\mathbf{r}^{\prime}\right|\right) \exp \left[-\lambda\left(z+z^{\prime}\right)\right] d \mathbf{x}^{\prime} / d q .
$$

An alternative expression for $P$ can be obtained by repeated application of Stokes' theorem to formula (2.19):

$$
P(\lambda)=\int_{R} \int_{T} \lambda^{2} J_{0}\left(\lambda\left|\mathbf{r}-\mathbf{r}^{\prime}\right|\right) \exp \left[-\lambda\left(z+z^{\prime}\right)\right],
$$

where the integrations are now over the areas of the loops. This form for $P$ is generally more convenient for calculation because it involves only scalar quantities. For example, when the transmitting and receiving loops are circular, with radii $a$ and $b$, and centres separated by a distance $d$, repeated application of the addition theorem for Bessel functions gives

$$
P(\lambda)=4 \pi^{2} a b J_{1}(\lambda a) J_{1}(\lambda b) J_{0}(\lambda d) .
$$

The TEM response of the layered medium is obtained from $Z(s)$ by inverse Laplace transform,

$$
Z(t)=(2 \pi i)^{-1} \int_{C} d s Z(s) \exp (s t)
$$


where $C$ is a contour parallel to the imaginary $s$ axis and which lies entirely to the right of the singularities of $Z(s)$. Our strategy is to locate these singularities and deform the contour about them in such a way that Watson's lemma can be applied to the integral representation of $Z(t)$.

\section{Singuiarities of $Z(s)$}

We observe that $s I(s)$ is an entire function of $s$, namely,

$$
s I(s)=[1-\exp (-s \tau)] /(s \tau),
$$

so we can focus upon the functions $a$ and $b$ which appear in the matrix $A$.

Each factor $T$ which appears in the expansion of $A$ is an entire function of

$$
k_{i}^{2}=\varepsilon_{i} s^{2}+\sigma_{i} s+\lambda^{2}, \quad 1 \leqslant i \leqslant n,
$$

and so is necessarily entire in both $s$ and $\lambda$. Consequently $A$ is a holomorphic function of $s$ except when

$$
k_{0}^{2}=\varepsilon_{0} s^{2}+\lambda^{2} \leqslant 0
$$

or

$$
k_{n+1}^{2}=\varepsilon_{n+1} s^{2}+\sigma_{n+1} s+\lambda^{2} \leqslant 0 .
$$

A short analysis shows that, for any fixed $\lambda, A$ is holomorphic in the variable $s$ throughout the plane cut as shown in Figure 3. Note that the endpoints shown for the cut on the real axis are only correct asymptotically as $\varepsilon_{n+1} \rightarrow 0$.

Let us compute the discontinuity of $b / a$ across the cut on the real axis. To do so we establish the convention that the value of $k_{i}$ on its associated cut is taken to be its boundary value from above. Let

$$
s=u+i v,
$$

and define for $u$ on the cut

$$
D(u)=(2 \pi i)^{-1} \lim _{V \rightarrow 0^{+}}[(b / a-p)(u-i v)-(b / a-p)(u+i v)] .
$$

Since the sign of $k_{n+1}$ reverses when $s$ crosses the cut, and since

$$
\begin{aligned}
& b\left(-k_{n+1}\right)=d\left(k_{n+1}\right), \\
& a\left(-k_{n+1}\right)=c\left(k_{n+1}\right),
\end{aligned}
$$

we find that

$$
\begin{aligned}
D(u) & =(2 \pi i)^{-1} \lim _{v \rightarrow 0^{+}}[d / c-b / a](u+i v) \\
& =(2 \pi i)^{-1} \lim _{v \rightarrow 0^{+}}[(a d-b c) /(a c)](u+i v) .
\end{aligned}
$$




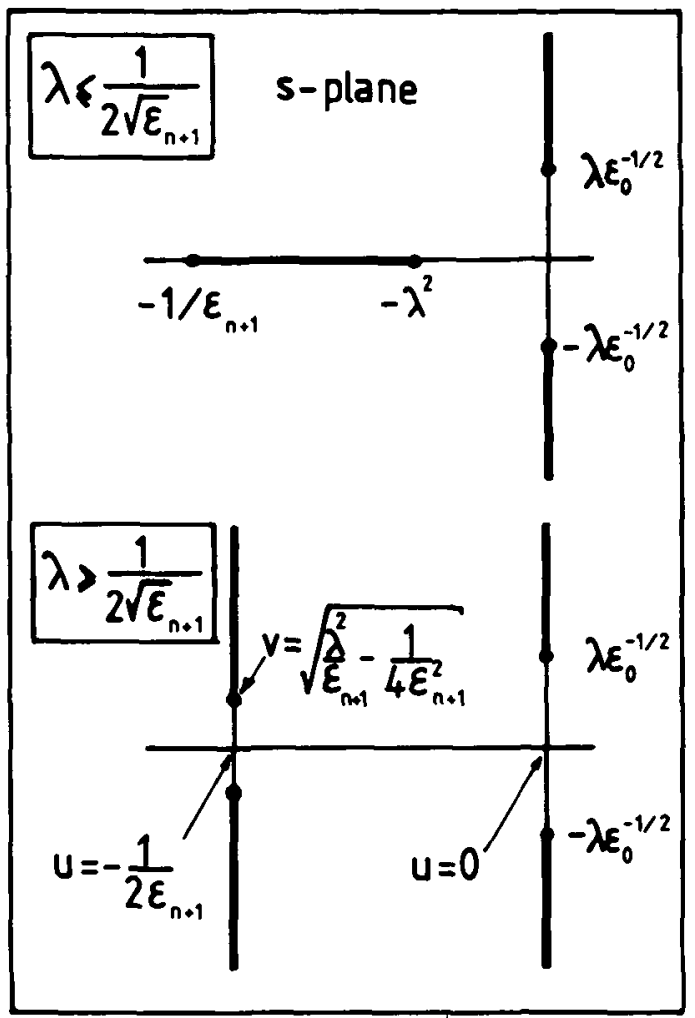

FIGURE 3

Now

$$
a d-b c=\operatorname{det} A=k_{n+1} / k_{0},
$$

so

$$
D(u)=k_{n+1} /\left(2 \pi i k_{0} a c\right) \text {, }
$$

where the limit sign has been omitted on the understanding that all quantities are defined on the cut by their boundary values from above.

The analytic structure of $A$ simplifies if we now introduce the quasistatic approximation which sets

$$
\varepsilon_{i}=0, \quad i=0,1, \ldots, n+1 .
$$

The cuts parallel to the imaginary axis recede to infinity, as does the left hand endpoint of the cut on the real axis. Thus, for fixed $\lambda, A$ is holomorphic in the whole $s$ plane, with the exception of a cut along the real axis from infinity to $-\lambda^{2}$. The discontinuity of $b / a$ across this cut is

$$
D(u)=\left(\lambda^{2}+u\right)^{1 / 2} /(2 \pi i \lambda a c)
$$


since

$$
k_{0}=\lambda
$$

and

$$
k_{n+1}=\left(\lambda^{2}+u\right)^{1 / 2}
$$

in the quasistatic approximation.

The quasistatic approximation is nearly always used in the analysis of geoprospecting systems because observations are made long after the wave fronts have passed the observer. Under these circumstances, the fields evolve according to a diffusion equation rather than a wave equation. We will now keep the quasistatic approximation for the rest of the paper.

In addition to the singularities of $Z(s)$ associated with the branch cuts of $k_{0}$ and $k_{n+1}$, there will also be poles at the zeros of $a$. Here we regard $a$ as a function of $s$ with parameter $\lambda$, and we denote the number of zeros by $n(\lambda)$ and their positions by $s_{1}(\lambda), \ldots, s_{n(\lambda)}(\lambda)$. These zeros correspond to eigenvalues of the operator

$$
L=-d^{2} / d z^{2}+q,
$$

where

$$
q=\varepsilon s^{2}+\sigma s,
$$

on the Sobolev space $H^{2}(\mathbf{R})$. That this should be so emerges naturally from the construction of Green's function $g$ along the lines sketched at the beginning of Section 1, but since most of the steps are fairly routine and would require us to introduce extensive notation, we will omit them here. Instead, we will show that the zeros must lie in the interval $\left[-\lambda^{2}, 0\right]$, and that $n(\lambda)$ is zero if $\lambda$ is sufficiently small.

From the positivity of the operator $-d^{2} / d z^{2}$, it is easy to show that

$$
\begin{array}{r}
\left(u^{2}-v^{2}\right)(f, \varepsilon f)+u(f, \sigma f)+\lambda^{2} \leqslant 0, \\
v[2 u(f, \varepsilon f)+(f, \sigma f)]=0,
\end{array}
$$

where $s=u+i v$ and $f$ is the normalised eigenfunction corresponding to the zero of $a$. In the quasistatic approximation, these inequalities reduce to

$$
\begin{aligned}
u(f, \sigma f)+\lambda^{2} & \leqslant 0, \\
v(f, \sigma f) & =0 .
\end{aligned}
$$

From (3.18) and (3.19) we conclude that the zeros of $a$ lie on the negative real axis. Since the point $-\lambda^{2}$ will lie in the continuous spectrum of $L$ if $s$ is in the interval $\left(-\infty,-\lambda^{2}\right]$, and since $L$ cannot have eigenvalues embedded in its continuous spectrum, it follows that the zeros of $a$ are confined to the interval $\left[-\lambda^{2}, 0\right]$. 
We obtain an upper bound on the zeros of $a$ by comparing the spectrum of $L$ with the spectrum of a related operator $L_{*}$, whose conductivity function has only a single layer above the basement.

LemMa. Let $\overline{\boldsymbol{\sigma}}$ denote the maximum value of $\sigma$, and suppose that $\bar{\sigma}$ is greater than 1. Then $n(\lambda)$ is zero if

$$
\lambda^{2}<M=\pi^{2} /\left[4 d^{2}(\bar{\sigma}-1)\right] .
$$

Any zeros of a must satisfy

$$
s_{i}(\lambda)<-M
$$

Proof. We will restrict $s$ to be real and negative, and show that, when $|s|$ is less than $M, L$ cannot have any eigenvalues. Consequently, $a$ cannot have any zeros. Equivalently, any zeros of $a$ must satisfy the inequality (3.21). Since all zeros of $a$ lie in the interval $\left[-\lambda^{2}, 0\right]$, then $|s|<M$ whenever (3.20) is true, so $n(\lambda)$ must be zero.

Let $q_{*}$ denote the 'potential' with $\sigma$ replaced by the function

$$
\sigma_{*}(z)= \begin{cases}1, & z<-d \\ \bar{\sigma}, & -d \leqslant z \leqslant 0 \\ 0, & 0<z\end{cases}
$$

and let $L_{*}$ denote the differential operator with $q$ replaced by $q_{*}$. Then $L$ and $L_{*}$ have the same essential spectrum, namely, the interval $[s, \infty$ ) (Schechter [18], Theorem 5.8.1), and, if $L_{*}$ does not have any eigenvalues, then neither can $L$. Indeed, if $L$ has an eigenvalue $\zeta$ below the essential spectrum, with normalised eigenfunction $f$, then

$$
\left(f, L_{*} f\right)+\left(f,\left[q-q_{*}\right] f\right)=\zeta<s .
$$

Since $q-q_{*}$ is both bounded and positive, it follows that

$$
\left(f, L_{*} f\right) \leqslant \zeta<s \text {, }
$$

which implies that $L_{*}$ also has an eigenvalue (Schechter [18], Corollary 4.4.1), and we have a contradiction. Lastly, it is a standard result from quantum mechanics that $L_{*}$ does not have any eigenvalues if $|s|<M$ (Schechter [18], Corollary 3.3.1).

Finally, we note the special case of a resistive overburden, for which

$$
\sigma(z) \leqslant 1 \text { for } z \leqslant 0 .
$$

Then

$$
(f, \sigma) \leqslant 1
$$

and so (3.18) implies that

$$
u \leqslant-\lambda^{2}
$$


This is a contradiction and so the function $a$ cannot have any zeros if the overburden is resistive.

The conclusion of the analysis is that the singularities of $b / a$ consist of a square-root branch point at the point $-\lambda^{2}$ and a finite number of simple poles in the segment $\left[-\lambda^{2}, 0\right]$. The branch cut for the square root lies along the segment $\left(-\infty,-\lambda^{2}\right]$ of the negative real axis. Consequently, the contour $C$ for the inverse Laplace transform can be any vertical line in the right hand $s$ plane.

In the next section we will deform the contour $C$ around the negative real axis of the $s$ plane. It is interesting to note here the role of the quasistatic approximation, without which the three cuts would pinch on the origin of the $s$ plane as $\lambda \rightarrow 0$. Whether or not the contour $C$ could be deformed would become quite a delicate question.

\section{Derivation of $Z(t)$}

The TEM response of the layered medium is

$$
Z(t)=-\left(8 \pi^{2} i\right)^{-1} \int_{C} d s \exp (s t) s I(s) \int_{0}^{\infty} d \lambda P(\lambda)[b / a-p](\lambda, s) .
$$

With the contour $C$ in the right half plane, we may interchange the order of integration. Having done so, we deform the contour $C$ around the negative real axis. Then

$$
\begin{aligned}
Z(t)=-\left(8 \pi^{2} i\right)^{-1} \int_{0}^{\infty} d \lambda & P(\lambda) \\
& \cdot\left\{\int_{-\infty}^{-\lambda^{2}} d s \exp (s t) s I(s)\right. \\
& \quad \lim _{v \rightarrow 0^{+}}[[b / a-p](u-i v)-[b / a-p](u+i v)] \\
& \left.\quad+(2 \pi i) \sum_{i=1}^{n(\lambda)} \underset{s=s_{1}(\lambda)}{\text { residue }}[\exp (s t) s I(s)(b / a-p)]\right\} .
\end{aligned}
$$

Because the zeros $s_{t}(\lambda)$ are strictly negative for all $\lambda$ and are bounded away from zero, it follows that the contributions of the pole terms are all exponentially damped in time. In fact, the second term in (4.2) is bounded by

$$
(4 \pi)^{-1} \exp (-M t) \int_{0}^{\infty} d \lambda\left|P(\lambda) \sum_{i=1}^{n(\lambda)} \underset{s=s_{i}(\lambda)}{\operatorname{residue}}[s I(s)(b / a-p)]\right| .
$$


Since we are interested in late times, we discard these terms and obtain

$$
Z(t)=-(4 \pi)^{-1} \int_{0}^{\infty} d \lambda P(\lambda) \int_{-\infty}^{-\lambda^{2}} d s \exp (s t) s I(s) D(s) .
$$

Reverse the order of integration again and substitute

$$
s=-x^{2} \text { and } \lambda=m x .
$$

Then

$$
Z(t)=\left[B_{1}(t)-B_{1}(t-\tau)\right] / \tau,
$$

where

$$
\begin{gathered}
B_{i}(t)=\pi^{-2} \int_{0}^{\infty} d x \exp \left(-x^{2} t\right) x^{2(t-1)} S(x), \\
S(x)=x^{2} \int_{0}^{1} d m\left(1-m^{2}\right)^{1 / 2} m P(m x) / Q(m, x),
\end{gathered}
$$

and

$$
Q(m, x)=4 \lambda^{2} a c .
$$

In the limit as $\tau \rightarrow 0$, we find

$$
Z(t)=-B_{2}(t)
$$

\section{Asymptotic analysis of $B_{t}(t)$}

We now apply Watson's lemma (Copson [4]) to (4.6) to obtain an asymptotic expansion for $B_{i}(t)$ at late times. We develop $S$ as a convergent power series for small $x$,

$$
S(x)=x^{2} \sum_{r=0}^{\infty} S_{r} x^{r}
$$

substitute this into (4.6) and integrate termwise to obtain

$$
B_{i}(t) \sim A_{i}(t)=\left(2 \pi^{2}\right)^{-1} \sum_{r=0}^{\infty} S_{r} \Gamma((r+1) / 2+i) t^{-((r+1) / 2+i)} .
$$

In this section we will develop two expressions for the Taylor coefficients $S_{r}$, the first of which will be a convenient tool for the analysis but unsuited to numerical computation, whereas the second will have the opposite properties.

The first step is to develop $Q$ in power series for small $x$, which we achieve by expanding the factors in the matrix $A$ in series and then multiplying the series together. Next we develop $P$ in series and compute the ratio $P / Q$. Finally we integrate over $m$. 
The matrix $T_{r}$ has the series representation

$$
T_{r}=\sum_{i_{r}=0}^{\infty} \frac{\left(k_{r} d_{r}\right)^{2 i_{r}}}{\left(2 i_{r}\right) !} T_{r}^{i_{r}},
$$

where

$$
T_{r}^{i_{r}}=\left[\begin{array}{cc}
1 & 2 i_{r} / d_{r} \\
a_{r} /\left(2 i_{r}+1\right) & 1
\end{array}\right] .
$$

If we let $X=T_{n} T_{n-1} \cdots T_{1}$, then

$$
X=\sum_{r=0}^{\infty} X_{r} x^{2 r}
$$

where

$$
\begin{gathered}
X_{r}=\sum_{|l|=r}\left(m^{2}-\sigma_{1}\right)^{i_{1}} \cdots\left(m^{2}-\sigma_{n}\right)^{i_{n}} X_{r}^{\prime}, \\
X_{r}^{i}=\frac{d_{1}^{2 l_{1}} \cdots d_{n}^{2 i_{n}}}{\left(2 i_{1}\right) ! \cdots\left(2 i_{n}\right) !} T_{n}^{i_{n}} \cdots T_{1}^{l_{1}},
\end{gathered}
$$

and

$$
\begin{aligned}
i & =\left(i_{1}, \ldots, i_{n}\right), \\
|i| & =i_{1}+\cdots+i_{n} .
\end{aligned}
$$

Let

$$
X=\left[\begin{array}{ll}
\alpha & \beta \\
\gamma & \delta
\end{array}\right], \quad X_{r}=\left[\begin{array}{cc}
\alpha_{r} & \beta_{r} \\
\gamma_{r} & \delta_{r}
\end{array}\right] .
$$

A short calculation yields that

$$
Q=(\alpha \lambda+\beta)^{2}+x^{2}\left(1-m^{2}\right)(\gamma \lambda+\delta)^{2},
$$

so we obtain the series for $Q$ by further multiplication.

$$
\begin{aligned}
& (\alpha \lambda+\beta)=m \sum_{r=0}^{\infty} \alpha_{r} x^{2 r+1}+\sum_{r=0}^{\infty} \beta_{r} x^{2 r}=\sum_{r=0}^{\infty} \mu_{r} x^{r+1}, \\
& (\gamma \lambda+\delta)=m \sum_{r=0}^{\infty} \gamma_{r} x^{2 r+1}+\sum_{r=0}^{\infty} \delta_{r} x^{2 r}=\sum_{r=0}^{\infty} \nu_{r} x^{r}
\end{aligned}
$$

where

$$
\begin{array}{ll}
\mu_{2 r}=m \alpha_{r}, & \nu_{2 r+1}=m \gamma_{r}, \\
\mu_{2 r-1}=\beta_{r}, & \nu_{2 r}=\delta_{r} .
\end{array}
$$

Hence,

$$
Q(m, x)=x^{2} \sum_{r=0}^{\infty} Q_{r} x^{r}
$$


where

$$
Q_{r}=\sum_{p+q=r}\left[\mu_{p} \mu_{q}+\left(1-m^{2}\right) \nu_{p} \nu_{q}\right] .
$$

Note that $Q_{0}=1$ and hence is independent of $m$. Since $X_{r}$ is a polynomial in $m$ of degree $2 r$, it follows trivially that

$$
\begin{array}{ll}
\text { degree }\left(\mu_{2 r}\right)=2 r+1, & \text { degree }\left(\nu_{2 r}\right)=2 r, \\
\text { degree }\left(\mu_{2 r-1}\right)=2 r, & \text { degree }\left(\nu_{2 r+1}\right)=2 r+1,
\end{array}
$$

and hence that the degree of $Q_{r}$ does not exceed $r+2$. In fact,

$$
\operatorname{degree}\left(Q_{r}\right)=r \text {. }
$$

To prove this, firstly observe that $\alpha_{r}, \beta_{r}, \gamma_{r}$ and $\delta_{r}$ are polynomials in $m^{2}$, and then establish by induction on $r$ that $Q_{2 r}$ and $m^{-1} Q_{2 r+1}$ must also be polynomials in $m^{2}$. Thus, $Q_{r}$ will have degree $r$ if the coefficient of $m^{r+2}$ is zero. But this coefficient is independent of $\sigma_{1}, \ldots, \sigma_{r}$, so we set

$$
\sigma_{1}=\sigma_{2}=\cdots=\sigma_{n}=1
$$

and find that

$$
Q(m, x)=x^{2},
$$

which clearly demonstrates that the coefficient of $m^{r+2}$ is always zero if $r>0$.

It is quite apparent that formulae (5.6) and (5.7) are unsuited to numerical computation, because the number of terms in the summation for $X_{r}$ becomes extremely large for more than two layers and the cost of computing the partitions of $r$ and the matrix products is excessive. Fortunately, it is not difficult to prove by induction that

$$
X=\sum_{\varepsilon_{2}, \ldots, \varepsilon_{n}} C_{e}\left[\begin{array}{cc}
\cosh B_{e} & k_{1} \sinh B_{\varepsilon} \\
\left(\varepsilon_{n} k_{n}\right)^{-1} \sinh B_{e} & k_{1}\left(\varepsilon_{n} k_{n}\right)^{-1} \cosh B_{e}
\end{array}\right]
$$

where

$$
\begin{aligned}
& B_{\varepsilon}=k_{1} d_{1}+\varepsilon_{2} k_{2} d_{2}+\cdots+\varepsilon_{n} k_{n} d_{n}, \\
& C_{\varepsilon}=2^{1-n}\left(1+\varepsilon_{2} k_{2} / k_{1}\right)\left(1+\varepsilon_{3} k_{3} / \varepsilon_{2} k_{2}\right) \cdots\left(1+\varepsilon_{n} k_{n} / \varepsilon_{n-1} k_{n-1}\right) .
\end{aligned}
$$

The summation is over the signs $\varepsilon_{i}= \pm$, so the number of terms in the summation is $2^{n-1}$.

The ratios $k_{t+1} / k_{i}$ which appear in $C_{\varepsilon}$ are independent of $x$, so it is only necessary to expand the hyperbolic functions in order to obtain the series development of $X$. A short calculation gives

$$
X=\sum_{r=0}^{\infty} x^{2 r} \sum_{e} X_{r}^{(e)}
$$


where

$$
X_{r}^{(e)}=\frac{C_{e} K_{e}^{2 r}}{(2 r) !}\left[\begin{array}{cc}
1 & 2 r p_{1} / K_{e} \\
K_{e} /\left(\varepsilon_{n} p_{n}(2 r+1)\right) & p_{1} /\left(\varepsilon_{n} p_{n}\right)
\end{array}\right]
$$

and

$$
\begin{aligned}
p_{i} & =\left(m^{2}-\sigma_{i}\right)^{1 / 2}, \\
K_{e} & =p_{1} d_{1}+\varepsilon_{2} p_{2} d_{2}+\cdots+\varepsilon_{n} p_{n} d_{n} .
\end{aligned}
$$

Note that the earlier expression for $X_{r}$ indicated that its elements were polynomials in $m$, but the present formula does not even show clearly that the elements are real! Nonetheless, formula (5.18) is well suited for computation for the following reason. Let

$$
X_{r}^{(\varepsilon)}=\left[\begin{array}{ll}
\alpha_{r}^{(\varepsilon)} & \beta_{r}^{(\varepsilon)} \\
\gamma_{r}^{(e)} & \delta_{r}^{(\varepsilon)}
\end{array}\right],
$$

and define

$$
\begin{array}{ll}
\mu_{2 r}^{(e)}=m \alpha_{r}^{(e)}, & \nu_{2 r+1}^{(e)}=m \gamma_{r}^{(e)}, \\
\mu_{2 r-1}^{(e)}=\beta_{r}^{(e)}, & \nu_{2 r}^{(e)}=\delta_{r}^{(e)},
\end{array}
$$

so that

$$
\mu_{r}=\sum_{\varepsilon} \mu_{r}^{(\varepsilon)}, \quad \nu_{r}=\sum_{\varepsilon} \nu_{r}^{(\varepsilon)} .
$$

Then $\mu_{r}^{(e)}$ and $\nu_{r}^{(e)}$ satisfy the following recursion formula,

$$
\begin{aligned}
& \mu_{r+2}^{(e)}=\mu_{r}^{(e)} K_{e}^{2} /[(r+1)(r+2)], \\
& \nu_{r+2}^{(e)}=\nu_{r}^{(e)} K_{e}^{2} /[(r+1)(r+2)],
\end{aligned}
$$

with the initial values

$$
\begin{array}{ll}
\mu_{0}^{(\varepsilon)}=m C_{\varepsilon}, & \nu_{0}^{(\varepsilon)}=C_{\varepsilon} p_{1} /\left(\varepsilon_{n} p_{n}\right), \\
\mu_{1}^{(\varepsilon)}=p_{1} C_{\varepsilon} K_{\varepsilon}, & \nu_{1}^{(\varepsilon)}=C_{\varepsilon} K_{\varepsilon} m /\left(\varepsilon_{n} p_{n}\right) .
\end{array}
$$

This recursion formula is easy to program and fast in execution.

The loop function is also an entire function and from the representation (2.20) it is clear that it has a series development of the form

$$
P(m x)=m^{2} x^{2} \sum_{r=0}^{\infty} P_{r}(m x)^{r} .
$$

Expressions for the coefficients $P_{r}$ are given for coincident circular loops and arbitrary rectangular loops in Appendix 1. 
Both $P$ and $Q$ have a zero of the second order at $x=0$, so provided that $x$ is less than the modulus of the closest nonzero zero of $Q$, the series for $P$ and $Q$ can be manipulated as follows. Let

$$
\begin{aligned}
F & =P / Q \\
& =m^{2} \sum_{r=0}^{\infty} F_{r} x^{r},
\end{aligned}
$$

where

$$
F_{0}=P_{0}
$$

and

$$
F_{r}=m^{r} P_{r}-\sum_{p=1}^{r} Q_{p} F_{r-p}, \quad r>0 .
$$

It is easy to prove by induction on $r$ that $F_{r}$ is a polynomial in $m$, that

$$
\text { degree }\left(F_{r}\right) \leqslant r \text {, }
$$

and that $F_{2 r}$ and $m^{-1} F_{2 r+1}$ are polynomials in $m^{2}$.

Within the region of convergence of the series (5.29), we may integrate termwise over $m$ to obtain

$$
S(x)=x^{2} \sum_{r=0}^{\infty} S_{r} x^{r}
$$

where

$$
S_{r}=\int_{0}^{1} d m\left(1-m^{2}\right)^{1 / 2} m^{3} F_{r}(m) .
$$

To evaluate $S_{r}$, two options are open.

(1) For odd orders, $F_{2 r+1}$ has the form

$$
F_{2 r+1}=m \cdot \text { polynomial in } m^{2} \text {. }
$$

Hence,

$$
S_{2 r+1}=2^{-1} \int_{-1}^{+1} d m\left(1-m^{2}\right)^{1 / 2} m^{3} F_{2 r+1}(m) .
$$

These integrals can be evaluated exactly by Gaussian quadrature with weight $\left(1-m^{2}\right)^{1 / 2}$, interval $[-1,+1]$, and Chebyshev polynomials of the second kind. (Stroud and Secrest [21]). To evaluate the even orders, let

$$
w=m^{2}
$$

and obtain

$$
S_{2 r}=2^{-1} \int_{0}^{1} d w(1-w)^{1 / 2} w F_{2 r}\left(w^{1 / 2}\right)
$$


Recall that $F_{2 r}\left(w^{1 / 2}\right)$ will be a polynomial in $w$. Thus, $S_{2 r}$ may be evaluated exactly by Gaussian quadrature with weight $(1-w)^{1 / 2}$, interval $[0,1]$ and Jacobi polynomials. (Krylov, Lugin and Yanovich [11].)

(2) Alternatively, quadrature rules may be developed for the interval $[0,1]$ with weight $\left(1-m^{2}\right)^{1 / 2} m^{3}$, so that both the odd and even order $S_{r}$ may be evaluated exactly with the same quadrature rule. This approach leads to a simpler program and is the one we have followed. We computed Gaussian rules with from one to twenty points. In order to compute $S_{r}$ exactly for

$$
0 \leqslant r \leqslant N=2 M-1 \text {, }
$$

we chose the rule with $M$ points:

$$
S_{r}=\sum_{i=1}^{M} w_{t} F_{r}\left(m_{l}\right),
$$

where $w_{t}$ and $m_{t}$ are the computed weights and nodes. In Appendix 2 are tabulated the weights and nodes for the 8 point rule, sufficient to give the first 16 terms of the asymptotic expansion.

The algorithm for computing the coefficients $S_{r}$ is summarised in the flow chart below.

Compute $P_{0}, P_{1}, \ldots, P_{N}$ for the loop configuration.

Set $S_{r}=0, r=0,1, \ldots, N$.

Set $m_{i}$ to the first quadrature node.

Label 1: Set $\mu_{r}=0$ and $\nu_{r}=0$.

Set $\varepsilon_{2}=+, \varepsilon_{3}=+, \ldots, \varepsilon_{n}=+$.

Label 2: Compute $B_{e}$ and $C_{e}$.

Compute $\mu_{0}^{(e)}, \mu_{1}^{(e)}$ and $\nu_{0}^{(e)}, \nu_{1}^{(e)}$.

Compute $\mu_{r}^{(e)}$ and $\nu_{r}^{(e)}$ by recursion.

Increment $\mu_{r}$ and $\nu_{r \cdot} \mu_{r}:=\mu_{r}+\mu_{r}^{(\varepsilon)}, \nu_{r}:=\nu_{r}+\nu_{r}^{(\varepsilon)}$

If not finished all sign combinations, go to 2 .

Compute $Q_{0}, Q_{1}, \ldots, Q_{n}$ from $\mu_{r}$ and $\nu_{r}$.

Compute $F_{0}, F_{1}, \ldots, F_{N}$ recursively from $P_{r}$ and $Q_{r}$.

Increment $S_{r} . S_{r}:=S_{r}+w_{i} F_{r}\left(m_{\imath}\right)$.

If not finished all quadrature points, go to 1 .

Stop.

\section{Thin layer approximations}

At this point we can establish the relation between our asymptotic expansion and the expansion to two terms obtained by Lee [13]. To do so, let

$$
Q^{(k)}=x^{2} \sum_{r=0}^{k} Q_{r} x^{r}
$$


and call $Q^{(k)}$ the $k^{\text {th }}$ thin layer approximation to $Q$. Since $X_{r}$ contains a factor $d_{1}^{2 i_{1}} \ldots d_{n}^{2 i_{n}}$, it is clear that the terms in the expansion of $X$ (and hence of $Q$ ) will become very small if the layers are thin, so it is reasonable to truncate the series as in (6.1) above. Explicit calculation yields

$$
\begin{aligned}
& Q_{0}=1, \\
& Q_{1}=2 m d \theta_{1}, \\
& Q_{2}=d^{2}\left[\left(\theta_{1}+\theta_{2}\right)\left(2 m^{2}-1\right)+\theta_{1}^{2}\right],
\end{aligned}
$$

where $\theta_{1}$ and $\theta_{2}$ are given by (1.14).

If the series for $Q$ is truncated at the first term, then the recurrence formula (5.31) degenerates to

$$
F_{r}=m^{r} P_{r}
$$

from which we obtain

$$
S_{r}=c_{r} P_{r},
$$

where

$$
\begin{aligned}
c_{r} & =\int_{0}^{1} d m\left(1-m^{2}\right)^{1 / 2} m^{3+r}, \\
& =\frac{\Gamma(3 / 2) \Gamma((r+4) / 2)}{2 \Gamma((r+7) / 2)} .
\end{aligned}
$$

Thus,

$$
A_{i}(t)=\left(2 \pi^{2}\right)^{-1} \sum_{r=0}^{\infty} c_{r} P_{r} \Gamma((r+1) / 2+i) t^{-((r+1) / 2+t)} .
$$

This is the response of a uniform half space with the conductivity of the basement, as expected, because all information concerning the layers was contained in the terms dropped from $Q$.

The first order thin layer approximation gives

$$
Q^{(1)}=x^{2}\left[1+2 m x d \theta_{1}\right] .
$$

Note that $Q^{(1)}$ can vanish if $\theta_{1}<0$, corresponding to a conductive overburden, so the thin layer approximation has introduced a spurious zero on the $x$ axis. The recurrence relation for $F_{r}$ can again be solved explicitly, because equation (5.31) reduces to

$$
\begin{aligned}
& F_{0}=P_{0}, \\
& F_{r}=m^{\prime} P_{r}-Q_{1} F_{r-1},
\end{aligned}
$$


which has the solution

$$
\begin{aligned}
F_{r} & =\sum_{p=0}^{r}\left(-Q_{1}\right)^{p} m^{r-p} P_{r-p} \\
& =m^{\prime} L_{r}
\end{aligned}
$$

where

$$
L_{r}=\sum_{p=0}^{r}\left(-2 d \theta_{1}\right)^{p} P_{r-p}
$$

Thus,

$$
S_{r}=c_{r} L_{r}
$$

and

$$
A_{i}(t)=\left(2 \pi^{2}\right)^{-1} \sum_{r=0}^{\infty} c_{r} L_{r} \Gamma((r+1) / 2+i) t^{-((r+1) / 2+i)} .
$$

Lee [13] obtained the first two terms of this asymptotic expansion for the case of coincident circular transmitting and receiving loops.

\section{Error estimates}

If the function $Q$ never vanished for nonzero $x$, then the series for $S$ would converge for all $x$ and the asymptotic expansion would be a convergent series. This happens only for the case of a uniform half space, for which

$$
Q=x^{2},
$$

so in principle the transient response of the half space could be calculated with arbitrary accuracy by summing sufficiently many terms of the series. (In practice, however, the series is so slowly convergent at early times that the finite word length of the computer causes large cancellation errors when many terms of the series are summed.) In all other cases, $Q$ is an entire function of order $\frac{1}{2}$, and so has an infinite number of isolated zeros. The proximity of these zeros to the origin limits the radius of convergence for the series for $S$, and hence reduces the range of times for which the asymptotic series is useful. For low conductivity contrasts or thin layers, the zeros of $Q$ are well away from the origin, and the asymptotic series 'converges' well, but, for larger conductivities and thicker layers, the zeros of $Q$ crowd in around the origin and the 'convergence' of the asymptotic series is poor. 
To make these intuitive ideas, precise we will develop an estimate for the error in chopping the asymptotic series at $N$ terms. The estimate will be in terms of the quantity

$$
\xi=\inf _{0 \leqslant m \leqslant 1} \xi(m)
$$

where

$$
\xi(m)=\inf _{k \geqslant 1}\left|x_{k}(m)\right|
$$

and

$$
0=x_{0}(m), x_{1}(m), x_{2}(m), \ldots
$$

are the distinct zeros of $Q$, regarded as a function of $x$ with parameter $m$. Clearly $\xi$ is the closest approach to the origin of all the zeros of $Q$ as $m$ is allowed to vary over the range $[0,1]$. In principle $\xi$ is computable, because $Q$ is an elementary combination of hyperbolic functions and its zeros can be found by a number of well established algorithms. However, the cost of such a search is not justified, firstly because the pragmatic algorithm works well, and secondly because we can approximate $\xi$ by the quantity $\xi^{(k)}$, defined analogously as the closest approach to the origin of the zeros of $Q^{(k)}$. It is easy to see that

$$
1 / \xi^{(1)}=2 d\left|\theta_{1}\right|
$$

and a rather lengthy, but straightforward, calculation yields

$$
1 / \xi^{(2)}= \begin{cases}d\left|\theta_{1}\right|\left(1+|\chi|^{1 / 2}\right), & x \leqslant 0, \\ d\left|\theta_{1}\right|(1+\chi)^{1 / 2}, & x \geqslant 0 .\end{cases}
$$

This is the approximation quoted in the introduction. Higher order approximations could be computed numerically, but again the cost is unwarranted. To illustrate this, the figures in Table 3 compare $\xi^{(1)}, \xi^{(2)}$ and $\xi$ in the case of a single conductive layer over a basement, for which $\xi$ can be computed exactly:

$$
\xi=\log \left[\left(\sigma_{1}^{1 / 2}+1\right) /\left(\sigma_{1}^{1 / 2}-1\right)\right] /\left(2 \sigma_{1}^{1 / 2} d_{1}\right) .
$$

TABLE 3

\begin{tabular}{|lcc|}
\hline & $\sigma_{1}=10, d_{1}=0.1$ & $\sigma_{1}=1000, d_{1}=1$ \\
\hline$\xi^{(1)}$ & 0.556 & $0.500 \times 10^{-3}$ \\
$\xi^{(2)}$ & 0.833 & $1.000 \times 10^{-3}$ \\
$\xi$ & 1.035 & $1.000 \times 10^{-3}$ \\
\hline
\end{tabular}

These figures also illustrate the general rule that the zeros crowd in around the origin as the product of conductivity and thickness increases. 
We now turn to the derivation of the error estimate. Let

$$
F^{N}=m^{2} \sum_{r=0}^{N} F_{r} x^{r}
$$

and

$$
S^{N}=x^{2} \int_{0}^{1} d m\left(1-m^{2}\right)^{1 / 2} m F^{N}(m) .
$$

LemMa. There exists a constant $K$ such that for all $x$

$$
\left|S(x)-S^{N}(x)\right|<K x^{3}(x / \xi)^{N} /|x-\xi| .
$$

Proof. For each $m, F$ has only simple poles, so the sequence

$$
\xi(m)^{r}\left|F_{r}(m)\right|, \quad r=0,1,2, \ldots,
$$

is bounded. Hence, there exists a constant $C(m)$ such that

$$
\xi(m)^{r}\left|F_{r}(m)\right|<C(m) \text { for all } r .
$$

Since $\xi<\xi(m)$ for all $m$, it follows that

$$
\left|F_{r}(m)\right|<C(m) / \xi^{r} \text { for all } r .
$$

Consider $x<\xi$. Since the series for $F$ is convergent for such $x$,

$$
S(x)-S^{N}(x)=x^{2} \int_{0}^{1} d m\left(1-m^{2}\right)^{1 / 2} m^{3} \sum_{r=N+1}^{\infty} F_{r}(m) x^{r},
$$

and

$$
\begin{aligned}
\left|S(x)-S^{N}(x)\right| & <x^{2} \int_{0}^{1} d m\left(1-m^{2}\right)^{1 / 2} m^{3} \sum_{r=N+1}^{\infty} C(m)(x / \xi)^{r} \\
& =x^{2} C(x / \xi)^{N+1} /(1-x / \xi)
\end{aligned}
$$

where

$$
C=\int_{0}^{1} d m\left(1-m^{2}\right)^{1 / 2} m^{3} C(m) .
$$

Thus, (7.7) holds with $K=C$.

Now consider $x>\xi$. The series is no longer convergent, but it is certainly true that

$$
\begin{aligned}
\left|S(x)-S^{N}(x)\right|< & x^{2} \int_{0}^{1} d m\left(1-m^{2}\right)^{1 / 2} m|F(m, x)| \\
& +x^{2} \int_{0}^{1} d m\left(1-m^{2}\right)^{1 / 2} m^{3} \sum_{r=0}^{N}\left|F_{r}(m)\right| x^{r}
\end{aligned}
$$

Since $\left|J_{0}\right| \leqslant 1$, it follows from (2.20) that

$$
|P(\lambda)| \leqslant \lambda^{2}|T||R|,
$$


where $|T|$ and $|R|$ denote the areas of the transmitting and receiving loops. The function

$$
\left(1-m^{2}\right)^{1 / 2} m^{3} x^{2} / Q(m, x)
$$

has at worst an integrable singularity in $m$, so there exists a constant $L$ such that

$$
\int_{0}^{1} d m\left(1-m^{2}\right)^{1 / 2} m|P(m x) / Q(m, x)| \leqslant L \quad \text { for all } x \geqslant \xi .
$$

Hence,

$$
\begin{aligned}
\left|S(x)-S^{N}(x)\right| & <x^{2} L+x^{2} \int_{0}^{1} d m\left(1-m^{2}\right)^{1 / 2} m^{3} C(m) \sum_{r=0}^{N}(x / \xi)^{r} \\
& =x^{2}\left[L+C\left(1-(x / \xi)^{N+1}\right) /(1-x / \xi)\right] .
\end{aligned}
$$

Choose $K$ so that

$$
L+C(x / \xi)^{N+1} /(x / \xi-1)<K(x / \xi)^{N+1} /(x / \xi-1),
$$

a condition which will be satisfied provided that

$$
K>C+L N^{N} /(N+1)^{N+1} .
$$

Then

$$
\left|S(x)-S^{N}(x)\right|<x^{2} K(x / \xi)^{N+1} /(x / \xi-1) .
$$

Again (7.7) holds and the proof is complete.

\section{Define}

$$
B_{1}^{N}(t)=\pi^{-2} \int_{0}^{\infty} d x \exp \left(-x^{2} t\right) x^{2(t-1)} S^{N}(x)
$$

Choose any small positive number $\varepsilon$, split the integration into three ranges, $[0, \xi-\varepsilon],[\xi-\varepsilon, \xi+\varepsilon]$ and $[\xi+\varepsilon, \infty)$, and use the estimate for $\left|S(x)-S^{N}(x)\right|$ in the first and last ranges.

$$
\begin{aligned}
\pi^{2}\left|B_{i}(t)-B_{i}^{N}(t)\right|< & \int_{0}^{\xi-\varepsilon} d x \exp \left(-x^{2} t\right) x^{2(i-1)} K x^{3}(x / \xi)^{N} /(\xi-x) \\
& +\int_{\xi-\varepsilon}^{\xi+\varepsilon} d x \exp \left(-x^{2} t\right) x^{2(i-1)}\left|S(x)-S^{N}(x)\right| \\
& +\int_{\xi+\varepsilon}^{\infty} d x \exp \left(-x^{2} t\right) x^{2(t-1)} K x^{3}(x / \xi)^{N} /(x-\xi) \\
< & \varepsilon^{-1} K \int_{0}^{\infty} d x \exp \left(-x^{2} t\right) x^{2 i+1}(x / \xi)^{N} \\
& +\int_{\xi-\varepsilon}^{\xi+\varepsilon} d x \exp \left(-x^{2} t\right) x^{2(i-1)}\left|S(x)-S^{N}(x)\right| .
\end{aligned}
$$


The first integral is trivial. Apply the mean value theorem to the second integral.

$$
\begin{aligned}
\pi^{2}\left|B_{i}(t)-B_{i}^{N}(t)\right|< & (2 \varepsilon)^{-1} K \xi^{2(t+1)} \Gamma(N / 2+i+1) /\left(\xi t^{1 / 2}\right)^{N+2 i+2} \\
& +2 \varepsilon \exp \left(-z^{2} t\right) z^{2(i-1)}\left|S(z)-S^{N}(z)\right|,
\end{aligned}
$$

where $z$ is a point in the interval $[\xi-\varepsilon, \xi+\varepsilon]$. Provided that $0<\varepsilon<\xi$ and also that $\xi$ is not too small, then the dominant term is the first. In any case, we can aiways increase the constant $K$ in order to obtain the following bound.

$$
\left|B_{i}(t)-B_{i}^{N}(t)\right|<\left(2 \pi^{2} \varepsilon\right)^{-1} K \xi^{2(i+1)} \Gamma(N / 2+i+1) /\left(\xi t^{1 / 2}\right)^{N+2 t+2} .
$$

This is the bound mentioned in the introduction.

\section{Appendix 1}

We assume that both the transmitting and receiving loops lie on the surface of the ground, so that $z=z^{\prime}=0$.

Consider firstly the case of concident circular transmitting and receiving loops with radius $a$. Formula (2.21) for the loop function reduces to

$$
P(\lambda)=\left[2 \pi a J_{1}(\lambda a)\right]^{2} .
$$

Insert the series for $J_{1}^{2}$ given in formula (9.1.14) of Abramowitz and Stegun [1] to obtain

$$
\begin{aligned}
& P_{2 r+1}=0, \\
& P_{2 r}=(2 \pi a)^{2} \frac{(-1)^{r}(a / 2)^{2 r+2}(2 r+2) !}{r ![(r+1) !]^{2}(r+2) !} .
\end{aligned}
$$

The sequence of $P_{r}$ is best computed by recursion, since

$$
P_{2 r+2}=-P_{2 r} a^{2}(2 r+3) /[2(r+1)(r+2)(r+3)],
$$

and

$$
P_{0}=\pi^{2} a^{4} \text {. }
$$

Now consider rectangular transmitting and receiving loops with sides parallel to the $x$ and $y$ axes. Suppose that the transmitting loop encloses the area

$$
\begin{aligned}
& a^{\prime} \leqslant x \leqslant b^{\prime}, \\
& c^{\prime} \leqslant y \leqslant d^{\prime}
\end{aligned}
$$

and that the receiving loop encloses the area

$$
\begin{aligned}
& a \leqslant x \leqslant b, \\
& c \leqslant y \leqslant d .
\end{aligned}
$$


Insert the series for $J_{0}$ (Abramowitz and Stegun [1], formula (9.1.10)) to obtain

$$
P(\lambda)=\lambda^{2} \sum_{r=0}^{\infty} P_{r} \lambda^{r}
$$

where

$$
\begin{aligned}
& P_{2 r+1}=0, \\
& P_{2 r}=(-1)^{r} V_{r} /\left[2^{2 r}(r !)^{2}\right]
\end{aligned}
$$

and

$$
U_{r}=\int_{R} \int_{T}\left|\mathbf{r}-\mathbf{r}^{\prime} s\right|^{2 r} .
$$

The integrations in (8.10) are over the areas of the loops. For the rectangular loops,

$$
V_{r}=\int_{a}^{b} d x \int_{c}^{d} d y \int_{a^{\prime}}^{b^{\prime}} d x^{\prime} \int_{c^{\prime}}^{d^{\prime}} d y^{\prime}\left[\left(x-x^{\prime}\right)^{2}+\left(y-y^{\prime}\right)^{2}\right]^{r}
$$

Use the binomial theorem on the power to reduce the integrand to a sum of multinomials which can then be integrated trivially. Thus,

$$
V_{r}=\sum_{k=0}^{r}\left(\begin{array}{l}
r \\
k
\end{array}\right) U_{k}\left(a, b, a^{\prime}, b^{\prime}\right) U_{r-k}\left(c, d, c^{\prime}, d^{\prime}\right)
$$

where

$U_{k}\left(a, b, a^{\prime}, b^{\prime}\right)=\frac{\left[\left(b-a^{\prime}\right)^{2 k+2}-\left(a-a^{\prime}\right)^{2 k+2}-\left(b-b^{\prime}\right)^{2 k+2}+\left(a-b^{\prime}\right)^{2 k+2}\right]}{[(2 k+1)(2 k+2)]}$.

Formulae (8.8), (8.12) and (8.13) give an easily computable expression for the sequence $P_{r}$.

\section{Appendix 2}

Listed in Table 4 are the points and weights for a Gaussian quadrature rule with weight $\left(1-m^{2}\right)^{1 / 2} m^{3}$ on the interval $(0,1)$.

TABLE 4

\begin{tabular}{|ccc|}
\hline & Points & Weights \\
\hline 1 & $0.908248748533967848 D+00$ & $0.278859654274717499 D-01$ \\
2 & $0.976532380300760677 D+00$ & $0.934191464302680789 D-02$ \\
3 & $0.801322605159335985 D+00$ & $0.379453158439124976 D-01$ \\
4 & $0.665445636715052751 D+00$ & $0.322020100441213207 D-01$ \\
5 & $0.51298670112516676 D+00$ & $0.180965420802834124 D-01$ \\
6 & $0.357914686098593863 D+00$ & $0.648895926666688655 D-02$ \\
7 & $0.214550949148259760 D+00$ & $0.128223423390867559 D-02$ \\
8 & $0.960799955038826807 D-01$ & $0.903917939419827435 D-04$ \\
\hline
\end{tabular}




\section{References}

[1] M.Abramowitz and I. A. Stegun, Handbook of mathematical functions (Dover, New York, 1965).

[2] W. L. Anderson, "Numerical integration of related Hankel transforms of orders 0 and 1 by adaptive digital filtering", Geophysics 44 (1979), 1287-1305.

[3] G. Buselli and B. O'Neill, "SIROTEM: A new portable instrument for multichannel transient electromagnetic measurements", ASEG Bull. 8 (1977), 82-87.

[4] E. T. Copson, Asymptotic expansions (Cambridge University Press, Cambridge, 1971).

[5] J. D. Crone, "Ground pulse EM-examples of survey results in the search for massive sulphides and new developments with the Crone PEM equipment", Paper presented by the author at the 25th international geological congress, Sydney, Australia (August, 1976).

[6] D. P. Gaver, Jr., "Observing stochastic processes and approximate transform inversion", Oper. Res. 14 (1966), 444-459.

[7] F. M. Kamenetski, "Transient processes using combined loops for a two layer section with a nonconducting base", Izv. Vuzov. Section Geology Prosp. 6 (1969), 108-113.

[8] A. A. Kaufman, "Harmonic and transient fields on the surface of a two layered medium", Geophysics 44 (1979), 1208-1217.

[9] J. H. Knight and A. P. Raiche, "Transient electromagnetic calculations using the Gaver-Stehfest inverse Laplace transform method", Geophysics 47 (1982), 47-50.

[10] O. Koefoed, D. P. Ghosh and G. J. Polman, "Computation of type curves for electromagnetic depth sounding with a horizontal coil by means of a digital linear filter", Geophys. Prosp. 20 (1972), 403-420.

[11] V. J. Krylov, V. V. Lugin and L. A. Yanovich, Tables for integration of functions with power singularities $\int_{0}^{1} x^{\beta}(1-x)^{\alpha} f(x) d x$ (Izdat. Akad. Nauk BSSR, Minsk, 1963).

[12] Y. L. Lamontagne, G. S. Lodha, J. C. Macnae and G. F. West, "UTEM: Wideband time domain EM project 1976-78, reports 1-5", Research in applied geophysics \#11, Geophysics laboratory, Department of Physics, University of Toronto (1980).

[13] T. Lee, "Asymptotic expansions for transient electromagnetic fields", Geophysics 47 (1982), $38-46$.

[14] T. Lee and R. Lewis, "Transient EM response of a large loop on a layered ground", Geophys. Prosp. 22 (1974), 430-444.

[15] K. Mallick and R. K. Verma, "Time domain electromagnetic sounding with horizontal and vertical coplanar loops on a multilayered earth", Geoexploration 16 (1978), 291-302.

[16] H. F. Morrison, R. J. Phillips and D. P. O'Brien, "Quantitative interpretation of transient electromagnetic fields over a layered half space", Geophys. Prosp. 17 (1968), 82-101.

[17] T. N. L. Patterson, "Algorithm for automatic numerical integration over a finite interval", Comm. ACM 16 (1973), 694-699.

[18] M. Schechter, Operator methods in quantum mechanics (North-Holland, New York, 1981).

[19] H. Stehfest, "Algorithm 368. Numerical inversion of Laplace transforms", Comm. ACM 13 (1970), 47-49.

[20] H. Stehfest, " Remark on algorithm 368", Comm. ACM 13 (1970), 624.

[21] A. H. Stroud and D. Secrest, Gaussian quadrature formulas (Prentice-Hall, Englewood Cliffs, N.J., 1966).

[22] J. R. Wait, Geoelectromagnetism (Academic Press, New York, 1982). 